\title{
Estimation of Lateral Motorcycle Dynamics and Rider Action with Luenberger Observer
}

\author{
P-M Damon, H. Dabladji, D. Ichalal, L. Nehaoua and H. Arioui
}

\begin{abstract}
This paper deals with lateral dynamics estimation of powered two-wheeled (PTW) vehicles. A linear parametervarying (LPV) model of the motorcycle is presented and then transformed into a Takagi-Sugeno (TS) form in order to design the observer under the variation of the longitudinal velocity. This paper presents a new way to estimate every motorcycle dynamic states including unknown input (UI) by keeping a simple observer structure with a Luenberger observer in TS form. The observer convergence study is based on the Lyapunov theory associated with LMI tools and $L_{2}$-gain to guaranty boundedness of the state estimation error. The effectiveness of the proposed solution is illustrated for a nominal and an uncertain simulation cases.
\end{abstract}

Index Terms-Motorcycle, Observation, LMI, Luenberger Observer, Augmented System, TS model, $L_{2}$-gain

\section{INTRODUCTION}

What if motorcycle could be an alternative to individual car transportation? Considering that the transport field represents $25 \%$ of greenhouse gases (GHG) emissions in France for the year 2014, solutions have to be figured out to reduce the use of individual car and improve traffic flow. By securing motorcycle travel, a renewed option could emerge by preserving the comfort of individual transportation while minimizing the ground space and the associated issues of congestion and emissions of GHG. Moreover, more and more PTW are equipped with electric powertrain making it free of carbon emission. Thus the number of single track vehicles is constantly increasing. Unfortunately, this expansion resulted in a growth of traffic fatalities. Statistics endorse this statement and riders are considered as the most vulnerable road users. In 2014, the French Agency of Road Safety made an official finding of around 660 deaths (24\% of global traffic fatalities), while the rate of motorcycles in the national traffic does not exceed $1.5 \%$ [1].

Many research projects as [2] or [3] are initiated to solve this issue in order to improve security through the development of preventive and active safety systems which consists for the main part to quantify the risk. This risk is calculated with dedicated functions which enable to detect dangerous situations such as excessive velocity in cornering, changes in road adhesion, etc. They directly depend on the vehicle dynamics motion. So it is necessary to identify pertinent and useful dynamic states to quantify the risk. During the last half century motorcycle modelling have been

This work is supported by National Agency of Research under the framework VIROLO++.

All the authors are with IBISC laboratory,
Université d'Evry Val d'Essonne, Evry,

largely studied. In the literature one can find well-known models: [4], [5] and one of the most used is the one given by Sharp in [6].

Two kind of models exist, one dedicated to the vehicle design which is generally complex and aims to be as possible in adequation with the real dynamic behaviour of the PTW. Often this model works out-of-line. For example the wellknown simulator program called BikeSim is based on the Sharp's model [7] where the motorcycle is divided into 8 different bodies allowing 16 degrees of freedom. BikeSim gives results of simulation very near from the reality under an important condition: user have to known all motorcycle static and dynamic parameters as the mass, the inertia of each body, the tire parameters,... In practice only the motorcycle maker have the whole of parameters. But the software proposed defined motorcycle configurations allowing to perform simulations. The second kind is the model derived to perform in-line simulation and is used to develop on-board semiactive and active safety systems for vehicle. It aims to be the best compromise between complexity and difference of computed results with the real dynamic behaviour in order to be implementable inside the vehicle without powerful computer platform.

To simulate vehicle dynamics the model needs measures. The definition of pertinent combination of measuring sensors of motorcycle dynamic states is inherent to prevent from massive instrumentation leading to overpriced new vehicles. Moreover according to common technologies on the market we have to consider the difficulty to measure some dynamic states as the rider torque and noisy measures can seriously compromise the results computed with the model. So the whole state vector and model input are impossible to measure thus the observation techniques have to be used to estimate the unmeasurable states.

As for motorcycle modelling lot of works have been conducted to estimate motorcycle dynamic states. Longitudinal dynamic estimation of PTW is discussed in [8] or [9], whereas lateral estimation is addressed in [5], [10], [11], [12], [13] and recently in [14]. Concerning lateral estimation most of cited papers consider restrictive assumptions which regarding riding motorcycle practices are not in adequation with the reality. This paper proposes a solution to design a very simple Luenberger observer in TS form which enables to estimate every lateral dynamic state of PTW including the unknown input. The designed observer takes into account the dependence of forward speed variation motion and guaranty estimation error boundedness. The observer is studied on stable motorcycle speed range without any control. 
This paper is organized as follow. Section 2 describes problem statement. Section 3 introduces the motorcycle dynamics model. Sections 4 deals with observer design. Section 5 shows simulation results. Finally, Section 6 presents our conclusions.

\section{Motivation}

Several works deal with the lateral motorcycle dynamics estimation as presented in the previous section but very few of them consider a variation of longitudinal speed. In [15] the author considers longitudinal velocity $v_{x}$ constant before decoupling the model and construct the observer. A restrictive speed range is then defined to guarantee reliable estimation. In [16] the variation of the forward speed is considered using an extended Kalman filter but simulations are performed for a constraint speed range.

Moreover to estimate dynamic states including rider torque which is an unknown input, authors use complex structures of observer sometimes associate with differentiator as in [15] where a high order sliding mode observer (HOSMO) is combined with derivative estimator. In [17] an adaptive observer is used. Proportional multiple integral (PMI) observers and Kalman filter as discussed in previous paragraph are also used for PTW lateral estimation.

None of them uses a simple structure of observer often easier to implement in the vehicle. That's why this paper deals with this topic and shows the ability in estimating simultaneous dynamic states and unknown input taking into account forward speed variation. This method does not need any assumption about unknown input except the existence of its first time derivative which is always satisfied because the rider torque is naturally bounded and can not be discontinuous. Moreover the designed nominal observer is naturally robust regarding modelling uncertainties.

\section{LINEARIZED MODEL OF MOTORCYCLE}

The present section is based on our previous works. For details, please refer to [5].

The study of the dynamics of motorcycle vehicles highlights two main modes of motion: in-plane mode representing longitudinal motion and out-of-plane mode, which describes the lateral dynamics when cornering. This last mode involves the roll inclination, the yaw rotation, and the steering and lateral motions of the bike. We consider here only the out-ofmode dynamics of the PTW. The coupling between the two modes is materialized by considering a variable longitudinal velocity that appears in the lateral dynamics. The motorcycle dynamic model given in [5] is linearized around the straightrunning trim trajectory and can be expressed by the following state space representation:

$$
\left\{\begin{array}{ccc}
\dot{\bar{x}} & = & \bar{A}\left(v_{x}\right) \bar{x}+\bar{B} \tau \\
\bar{y} & = & \bar{C} \bar{x}
\end{array}\right.
$$

Where $\bar{x}=\left[\phi, \delta, v_{y}, \dot{\psi}, \dot{\phi}, \dot{\delta}, F_{y f}, F_{y r}\right]^{T}$ denotes the state vector. For the sake of simplicity and clarity, the time variable $t$ is omitted and it assumes $\bar{x}=\bar{x}(t) . F_{y f}$ and $F_{y r}$ represent, respectively, the lateral forces of the front and rear tires introduced in the state space representing the tire relaxation. $\tau$ denotes the rider torque. $\bar{A}\left(v_{x}\right)$ is a parameter-varying matrix related to the forward velocity $v_{x}$, whereas $\bar{B}$ is a time-invariant vector. $\bar{y}$ is the vector of measures and $\bar{C}$ is the observation matrix. For more details please refer to the Appendix.

\section{A. Augmented model for observer design}

The state-space representation of the motorcycle (1) is then considered. To guaranty observability and observer design conditions the needed measurements are the yaw rate $\dot{\psi}$ and roll rate $\dot{\phi}$ (both are given by the central unit); the steering angle $\delta$ and steering rate $\dot{\delta}$ which may be obtained from an optical encoder. Finally with the appropriate output matrix $\bar{C}$ it comes $\bar{y}=[\delta, \dot{\psi}, \dot{\phi}, \dot{\delta}]^{T}$

Regarding the model (1) it is not possible to directly implement a Luenberger observer because there is no information about the dynamic of the model input $\tau$. Indeed the rider's torque applied on the handlebar is very difficult to measure with conventional devices. That's why several works consider the rider torque as unknown then they design an unknown input observer (UIO) to estimate the dynamic states. According to the kind of UIO used it is sometimes necessary to combine the observer with a model inversion as in [15] to get an estimation of the unknown input.

To keep as much as possible a simple structure and design of observer by avoiding the use of an UIO the system is augmented. Considering the input $\tau$ as a state and that its first time derivative $\dot{\tau}$ exists which is always satisfied because the rider torque is naturally bounded and can not be discontinuous. The system (1) can be transformed as follows:

$$
\left\{\begin{array}{c}
\dot{x}=\left[\begin{array}{cc}
\bar{A}\left(v_{x}\right) & \bar{B} \\
0 & 0
\end{array}\right] x+\left[\begin{array}{l}
0 \\
1
\end{array}\right] \dot{\tau} \\
y=c \\
{\left[\begin{array}{l}
\bar{C} \\
0
\end{array}\right] x}
\end{array}\right.
$$

with $x=[\bar{x}, \tau]^{T}$ and $y=\bar{y}$ which respectively denote the augmented state vector and the output vector. Note the system as expressed in (2) is an exact form considering that $\dot{\tau}$ exists.

For the next sections let us consider the following notations:

$A\left(v_{x}\right)=\left[\begin{array}{cc}\bar{A}\left(v_{x}\right) & \bar{B} \\ 0 & 0\end{array}\right], C=\left[\begin{array}{l}\bar{C} \\ 0\end{array}\right], F=\left[\begin{array}{l}0 \\ 1\end{array}\right], f=\dot{\tau}$

\section{B. Exact T-S model of the augmented model}

In order to express the LPV model (2) in T-S fuzzy structure, let us consider the nonlinearity $v_{x}$. It is important to note that the motorcycle is naturally a strongly unstable system that is why to keep the stability of the model, in open loop way, without considering control the study is reduced on a stable speed range. Consequently, the bounds of the premise variables are given by: $v_{x_{\text {min }}}=40 \mathrm{~km} / \mathrm{h}$ and $v_{x_{\max }}=110 \mathrm{~km} / \mathrm{h}$. 
By following the well-known sector nonlinearity approach [18] the model (2) can be exactly expressed as follows:

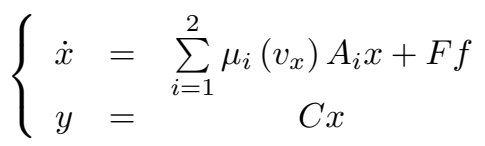

Note that there is one nonlinearity that's why the system is described with 2 sub-models. The variables $\mu_{i}($.$) are$ the weighing functions and they must satisfy the following convex sum property:

$$
\left\{\begin{array} { l } 
{ \sum _ { i = 1 } ^ { 2 } \mu _ { i } ( \nu ) = 1 } \\
{ 0 \leq \mu _ { i } ( \nu ) \leq 1 }
\end{array} \text { with } \left\{\begin{array}{l}
\mu_{1}=\frac{v_{x_{\max }}-v_{x}}{v_{x_{\max }}-v_{x_{\min }}} \\
\mu_{2}=\frac{v_{x}-v_{x_{\min }}}{v_{x_{\max }}-v_{x_{\min }}}
\end{array}\right.\right.
$$

The augmented model (3) expressed in exact TS form allows to design a Luenberger observer to estimate vehicle lateral dynamics and the rider torque.

\section{OBSERVER DESIGN}

In this section we explain the process to design the nominal Luenberger observer considering a general TS model. The approach considers Lyapunov theory associated with LMI tools and $L_{2}$-gain to guaranty ISS.

Let us consider the following TS model:

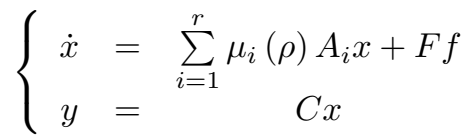

with $x \in \mathbb{R}^{n}, f \in \mathbb{R}^{n_{f}}$ and $y \in \mathbb{R}^{n_{y}}$ which are respectively the vector of states, the vector of perturbation and the vector of measures. $\rho(t) \in \mathbb{R}^{n_{\rho}}$ notes $\rho$ is the premise variable, $r$ is the number of sub-models given by $r=2^{l}$ with $l$ the number of nonlinearities and $\mu_{i}($.$) are the weighing$ functions and verifying the convex sum property (3) substituting 2 by $r$ sub-models. For the following observer design $\rho$ is considered measured.

Consider the well-known Luenberger observer in TS form:

$$
\left\{\begin{array}{llc}
\dot{\hat{x}} & = & \sum_{i=1}^{r} \mu_{i}(\rho)\left(A_{i} \hat{x}+L_{i}(y-\hat{y})\right) \\
\hat{y}= & C \hat{x}
\end{array}\right.
$$

with $L_{i}$ the observer gain matrices which ensure error convergence. The estimated state and output vector are respectively denoted $\hat{x}$ and $\hat{y}$. Now consider the state estimation error as follows:

$$
e=x-\hat{x}
$$

The dynamic of the error is given by:

$$
\begin{aligned}
\dot{e} & =\dot{x}-\dot{\hat{x}} \\
& =\sum_{i=1}^{r} \mu_{i}(\rho) A_{i} x+F f-\sum_{i=1}^{r} \mu_{i}(\rho)\left(A_{i} \hat{x}+L_{i}(y-\hat{y})\right) \\
& =\sum_{i=1}^{r} \mu_{i}(\rho) \mathcal{A}_{i} e+F f
\end{aligned}
$$

with $\mathcal{A}_{i}=A_{i}-L_{i} C$.
Finally, for stability analysis, one can consider the Lyapunov function $V$ with the symmetric definite positive matrix $X$ such that:

$$
V=e^{T} X e, \quad X=X^{T}>0
$$

whose time derivatives $\dot{V}$ leads to:

$\dot{V}=e^{T} \sum_{i=1}^{r} \mu_{i}(\rho)\left(\mathcal{A}_{i}^{T} X+X \mathcal{A}_{i}\right) e+f^{T} F^{T} X e+e^{T} X F f$

To attenuate the effect of the perturbation $f$ on the estimation error $e$ let us define the $L_{2}$-gain as the quantity:

$$
\sup _{\|f\|_{2} \neq 0} \frac{\|e\|_{2}}{\|f\|_{2}} \leq \gamma^{2}
$$

with $\gamma$ a positive scalar and $\|.\|_{2}$ the $L_{2}$-norm which for a vector $z(t)$ is given by:

$$
\|z(t)\|_{2}=\left(\int_{0}^{\infty} z^{T}(t) z(t) d t\right)^{1 / 2}
$$

The $L_{2}$-gain leads to the inequality:

$$
e^{T} e-\gamma^{2} f^{T} f<0
$$

Considering $\dot{V}<0$ it follows:

$$
\dot{V}+e^{T} e-\gamma^{2} f^{T} f<0
$$

By exploiting the expression of $\dot{V}$, inequality (14) can be expressed in matrix form as follows:

$$
\left[\begin{array}{l}
e \\
f
\end{array}\right]^{T}\left[\begin{array}{cc}
\sum_{i=1}^{r} \mu_{i}(\rho)\left(\mathcal{A}_{i}^{T} X+X \mathcal{A}_{i}\right)+I & X F \\
F^{T} X & -\gamma^{2}
\end{array}\right]\left[\begin{array}{l}
e \\
f
\end{array}\right]<0
$$

Since the weighting functions satisfy the convex sum property, sufficient conditions ensuring the convergence of estimation error are obtained as follows:

$$
\left[\begin{array}{cc}
\mathcal{A}_{i}^{T} X+X \mathcal{A}_{i}+I & X F \\
F^{T} X & -\gamma^{2}
\end{array}\right]<0, \quad i=1, \ldots, r
$$

In order to obtain solvable LMI conditions, the change of variables $\bar{L}_{i}=X L_{i}, A_{i}=\mathcal{A}_{i}+L_{i} C$ and $\bar{\gamma}=\gamma^{2}$ are performed which allows to obtain the LMI:

$\left[\begin{array}{cc}A_{i}^{T} X+X A_{i}-\bar{L}_{i} C-C^{T} \bar{L}_{i}^{T}+I & X F \\ F^{T} X & -\bar{\gamma}\end{array}\right]<0, \quad i=1, \ldots, r$

Finally, given a scalar $\gamma$, if there exists a symmetric and positive definite matrix $\mathrm{X}$ and matrices $\bar{L}_{i}, i=1, \ldots, r$ such that the LMI (17) is satisfied, then the error is stable and the transfer from the perturbation $f$ to the estimation error $e$ is bounded by $\gamma$. Note that the observer gain matrix $L$ is obtained by:

$$
L=\sum_{i=1}^{r} \mu_{i}(\rho) X^{-1} \bar{L}_{i}
$$

In practice to get better performance of the observer it is possible to transform the previous LMI (17) in an optimization problem by considering $\gamma$ as a variable parameter of optimization. 


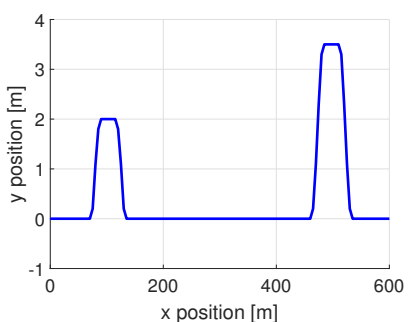

(a) Vehicle trajectory

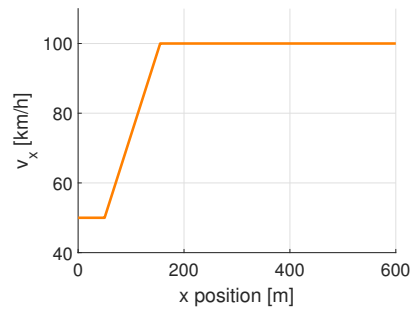

(b) Longitudinal velocity
Fig. 1: Overtaking and obstacle avoidance scenario

\section{Simulation Results}

\section{A. Nominal simulation}

In this section some simulations are provided to illustrate the effectiveness in estimating simultaneously the dynamic states and the rider torque on the LPV motorcycle model considering longitudinal velocity $v_{x}$ as time varying parameter. Motorcycle data used to perform simulations come from the simulator program BikeSim and are the same used in [15].

The scenario presented on figure 1 simulates two successive double lane changes. The first one with a lateral displacement at 2 meters while longitudinal speed increases uniformly from 50 to $100 \mathrm{~km} / \mathrm{h}$. The second with an amplified lateral displacement at 3,5 meters but the forward speed is considered constant speed at $100 \mathrm{~km} / \mathrm{h}$. Practically this scenario could represent an overtaking at constant acceleration followed with an obstacle avoidance at constant high speed.

BikeSim allows to simulate the whole of motorcycle dynamics including rider action which is considered as a regulator. That is why to get more realistic results taking into account rider control, the desired vehicle trajectory and longitudinal speed are implemented in the simulator to compute the resulted rider torque, the real longitudinal speed and vehicle trajectory. The considered outputs from BikeSim are then the inputs of our dynamic model and are given on figure 2 .

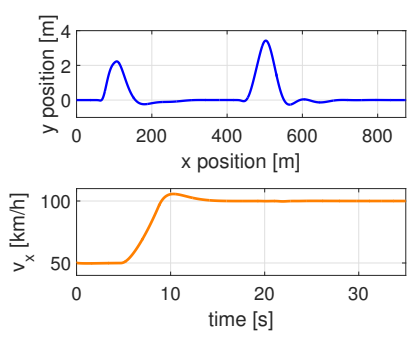

(a) Vehicle trajectory (blue) and longitudinal speed (orange)

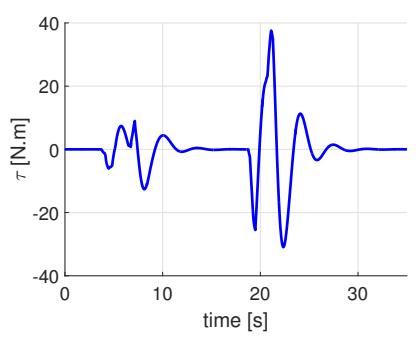

(b) Rider torque
Fig. 2: Overtaking and obstacle avoidance scenario simulate with BikeSim

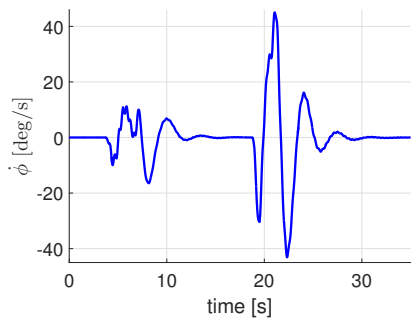

(a) Measured roll rate

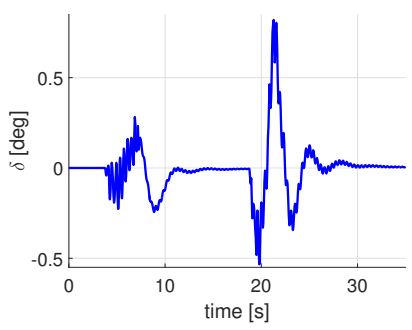

(c) Measured steering angle

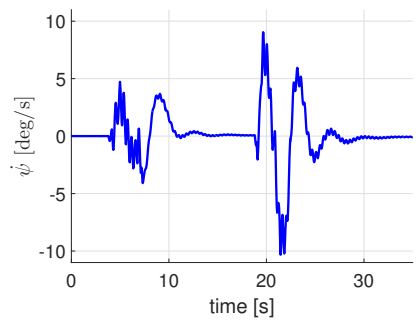

(b) Measured yaw rate

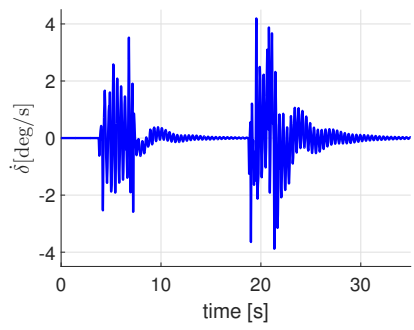

(d) Measured steering rate
Fig. 3: Measured states for nominal case

As discussed previously the measures are the yaw rate $\dot{\psi}$, the roll rate $\dot{\phi}$, the steering angle $\delta$ and the steering rate $\dot{\delta}$ which are respectively given by an inertial unit and an encoder for the steering mechanism angle and rate. Notice that these measures allow to estimate all dynamic states including rider torque. According to the simulation scenario described in figure 2, the measured states are given in the above figure 3 .

Then initial conditions of the observer are chosen different from the motorcycle model to show the transient phase of estimation error while the vehicle is considered in a straight running with null initial conditions at the beginning.

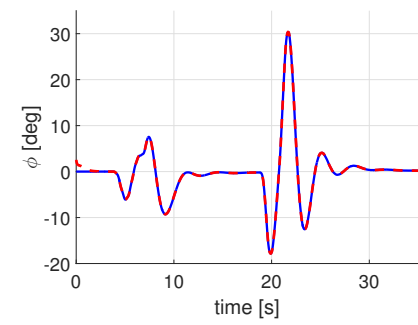

(a) Actual (blue) and estimated (red) roll angle

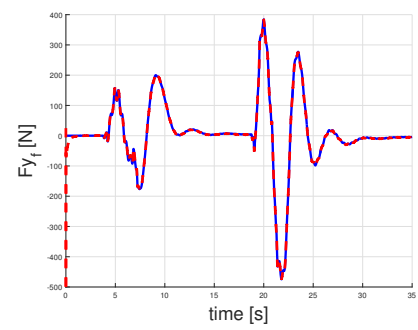

(c) Actual (blue) and estimated (red) front lateral tyre force

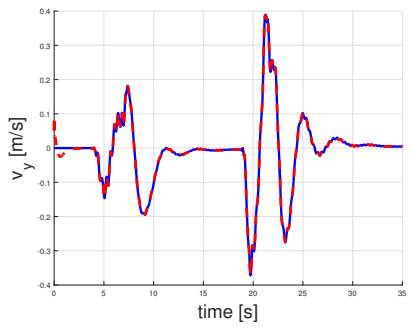

(b) Actual (blue) and estimated (red) lateral velocity

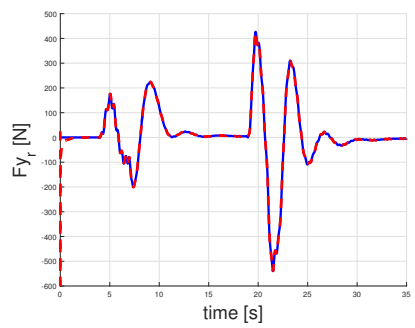

(d) Actual (blue) and estimated (red) rear lateral tyre force
Fig. 4: Estimated states for nominal case 


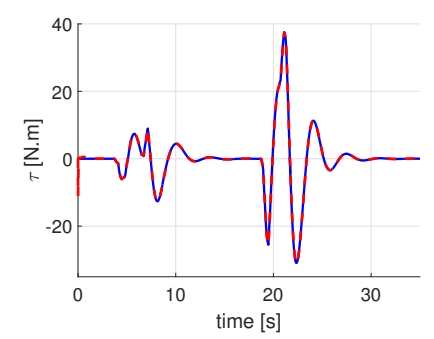

(e) Actual (blue) and estimated (red) rider's torque

Fig. 4: Estimated states for nominal case

We can see on figure 4 the non-measured states and rider torque with estimated states in red and actual states in blue which are perfectly estimated.

Finally, figure 4 shows the ability of the designed observer to perfectly recover the dynamics for a nominal case of simulation even if stability does not guaranty asymptotic convergence but bounded error convergence.

\section{B. Simulation with uncertainties}

Motorcycle model is highly dependant of many parameters as the inertia and mass of each body, the motorcycle geometry, etc. Usually the dynamic model is defined for a nominal case. A variation of some parameters inevitably leads to model uncertainties. The present section will focus on the rider weight uncertainty which is one of the most evident because every rider has a different morphology. The uncertainty inevitably impact the vehicle dynamics. Now let us consider a new rider with an uncertain weight of $30 \%$ from the nominal case, the system (2) becomes:

$$
\left\{\begin{array}{llc}
\dot{x} & = & \left(A\left(v_{x}\right)+\Delta A\right) x+F f \\
y & = & C x
\end{array}\right.
$$

with the model uncertainty noted $\Delta A$ which does not depend of the linear parameter varying $v_{x}$.

To validate the effects of model uncertainty on the present observer, a new scenario is defined in order to show a significant difference of the simulated dynamic states between nominal model (2) and uncertain model (19). Let us consider a scenario with a constant forward speed at $100 \mathrm{~km} / \mathrm{h}$ and a successive positive and negative torque step applied on the handlebar as it is shown on figure 5 .

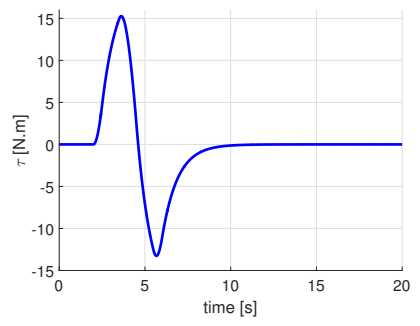

Fig. 5: Simulation scenario for uncertain case

Figure 6 shows the actual dynamic states compute with the nominal model in light blue and with the uncertain model in dark blue whereas estimated states are represented in red.

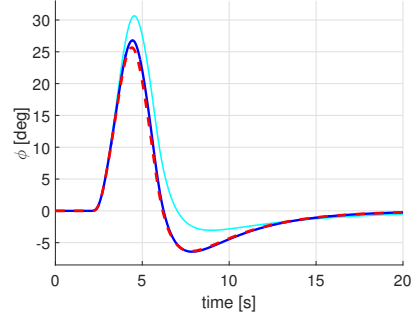

(a) Actual nominal (light blue), actual uncertain (dark blue) and estimated (red) roll angle

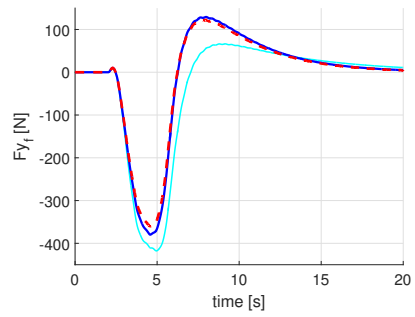

(c) Actual nominal (light blue), actual uncertain (dark blue) and estimated (red) front lateral tyre force

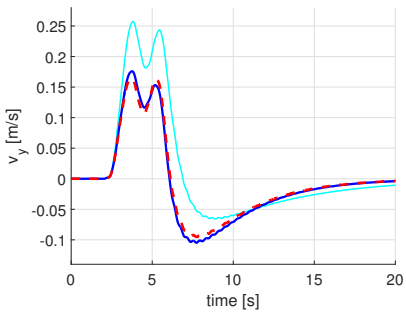

(b) Actual nominal (light blue), actual uncertain (dark blue) and estimated (red) lateral velocity

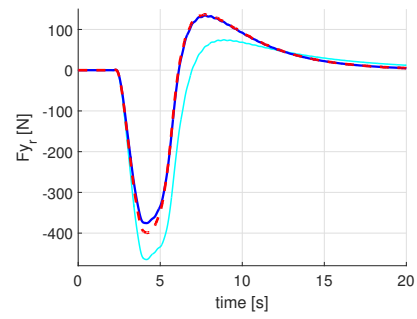

(d) Actual nominal (light blue), actual uncertain (dark blue) and estimated (red) rear lateral tyre force

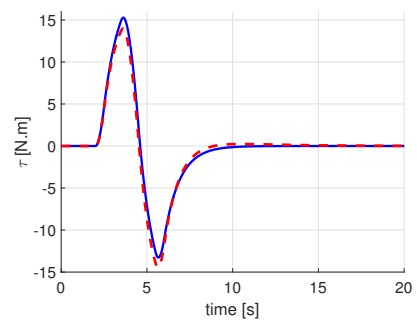

(e) Actual uncertain (dark blue) and estimated (red) rider torque

Fig. 6: Estimated states for uncertain case

Figure 7 shows the estimation error between estimated states and actual states obtained with nominal model in magenta and uncertain model in red.

Finally, the two figures 6 and 7 show that estimated states converge to actual states given by the uncertain model. This two last figures illustrate clearly the robustness of the proposed observer even if dynamic states are clearly affected by the uncertainties.

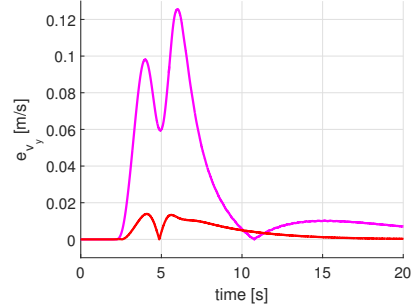

(a) Nominal (magenta) and uncertain (red) estimation error of lateral velocity

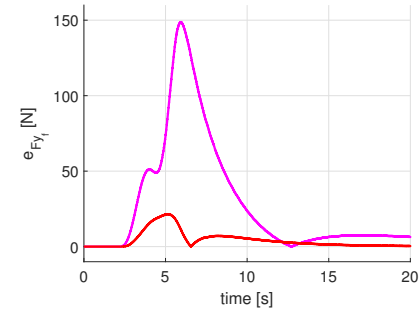

(b) Nominal (magenta) and uncertain (red) estimation error of front lateral tyre force
Fig. 7: State estimation errors for uncertain case 


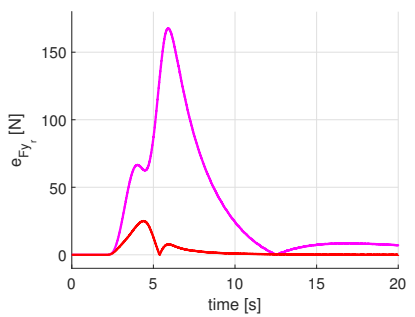

(c) Nominal (magenta) and uncertain (red) estimation error of rear lateral tyre force

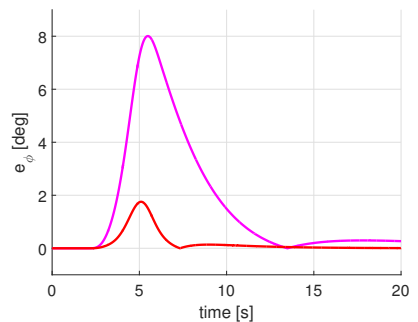

(d) Nominal (magenta) and uncertain (red) estimation error of roll angle
Fig. 7: State estimation errors for uncertain case

\section{CONCLUSION}

In this paper we have described the design process of a Luenberger observer in TS form to simultaneous estimate the lateral motorcycle dynamic states and the rider torque. The design method takes into account the forward speed as a linear parameter varying. The LPV model of the motorcycle used for observer synthesis is derived from Jourdain's principal and linearized around straight position. To conclude the design, observer stability analysis is addressed with Lyapunov theory associates with LMI tools and $L_{2}$-gain to guaranty ISS property. Then simulation are performed with a realistic riding scenario. First a nominal case is simulated, results are provided to show effectiveness of perfectly estimate the model state. Then uncertain model are consider with uncertainty on the rider weight. Results demonstrate that the proposed observer is naturally robust.

\section{APPENDIX}

$$
\begin{gathered}
\bar{A}\left(v_{x}\right)=\left[\begin{array}{cccccccc}
0 & 0 & 0 & 0 & 1 & 0 & 0 & 0 \\
0 & 0 & 0 & 0 & 0 & 1 & 0 & 0 \\
a_{31} & a_{32} & 0 & a_{34} & a_{35} & a_{36} & a_{37} & a_{38} \\
a_{41} & a_{42} & 0 & a_{44} & a_{45} & a_{46} & a_{47} & a_{48} \\
a_{51} & a_{52} & 0 & a_{54} & a_{55} & a_{56} & a_{57} & a_{58} \\
a_{61} & a_{62} & 0 & a_{64} & a_{65} & a_{66} & a_{67} & a_{68} \\
a_{71} & a_{72} & a_{73} & a_{74} & 0 & a_{76} & a_{77} & 0 \\
a_{81} & 0 & a_{83} & a_{84} & 0 & 0 & 0 & a_{88}
\end{array}\right] \\
\bar{B} \\
=\left[\begin{array}{lllllllll}
0 & 0 & b_{3} & b_{4} & b_{5} & b_{6} & 0 & 0
\end{array}\right]^{T}
\end{gathered}
$$

\footnotetext{
Numeric parameters $a_{i j}$ and $b_{i} \quad i, j=1 . .8$

$a_{31}=28.340, a_{32}=-0.780, a_{34}=-0.942 . v_{x}, a_{35}=$ $-0.017 . v_{x}, a_{36}=0.026 . v_{x}-0.351, a_{37}=0.009, a_{38}=$ $0.019, a_{41}=-3.209, a_{42}=-1.142, a_{44}=0.011 . v_{x}, a_{45}=$ $-0.007 . v_{x}, a_{46}=0.006 \cdot v_{x}+0.538, a_{47}=0.013, a_{48}=$ $-0.014, a_{51}=47.171, a_{52}=-1.170, a_{54}=0.095 . v_{x}$, $a_{55}=-0.059 . v_{x}, a_{56}=0.048 . v_{x}-0.636, a_{57}=0.013$, $a_{58}=0.022, a_{61}=-103.307, a_{62}=69.066, a_{64}=$ $-1.173 . v_{x}, a_{65}=-2.907 . v_{x}, a_{66}=0.0478 . v_{x}-25.529$, $a_{67}=-0.111, a_{68}=0.0455, a_{71}=-5281.460 . v_{x}$ $a_{72}=104503 \cdot v_{x}, a_{73}=-112042, a_{74}=-106440$, $a_{76}=5480.620, a_{77}=-5 . v_{x}, a_{81}=-2592.44 . v_{x}, a_{83}=$ $-88282.8, a_{84}=37078.4, a_{88}=-5 . v_{x}$

$b_{3}=0.028, b_{4}=-0.042, b_{5}=0.050, b_{6}=2.014$
}

\begin{tabular}{ll}
\hline Notations & \\
\hline$\dot{x}, \ddot{x}$ & time derivatives of the var $x$ \\
$\hat{x}$ & estimate of a variable $x$ \\
$x^{T}$ & transpose of vector or matrix $x$ \\
$x_{f}, x_{r}$ & denotes front and rear \\
& \\
\hline \multicolumn{2}{l}{ Variables and matrices } \\
\hline$v_{x}, v_{y}$ & longitudinal and lateral speeds \\
$\varphi, \psi, \delta$ & roll, yaw and steer angles \\
$\tau$ & rider torque \\
$F_{y_{f}}, F_{y_{r}}$ & lateral forces \\
$\bar{A}\left(v_{x}\right), A\left(v_{x}\right)$ & state matrices \\
$\bar{B}, B$ & input vectors \\
$\bar{C}, C$ & observation matrices
\end{tabular}

\section{REFERENCES}

[1] ONISR, "La sécurité routière en france: Bilan de l'accidentalité de l'année 2014," ONISR (Observatoire National Interministeriel de la Sécurité Routière), Tech. Rep., 2014.

[2] B. Evangelos, "Saferider project," French National Agency of Reasearch, Tech. Rep., 2010.

[3] N. L. Haworth, P. J. Rowden, D. E. Wishart, L. Buckley, K. Greig, and B. C. Watson, "Motorcycle rider safety project," The center for Accident Research \& Road Safety Queensland, Tech. Rep., 2012.

[4] H. Pacejka, Tire and Vehicle Dynamics. SAE International, 2005.

[5] L. Nehaoua, L. Nouvellière, and S. Mammar, "Dynamics modeling of a two-wheeled vehicle using Jourdain's principle," in 19th Mediterranean Conference on Control Automation (MED),, June 2011, pp. 1088-1093.

[6] R. S. Sharp, "The stability and control of motorcycles," Journal of Mechanical Engineering Science, vol. 13, no. 5, pp. 316-329, 1971.

[7] R. Sharp, S. Evangelou, and D. J. Limebeer, "Advances in the modelling of motorcycle dynamics," Multibody system dynamics, vol. 12, no. 3, pp. 251-283, 2004.

[8] M. E.-H. Dabladji, D. Ichalal, H. Arioui, and S. Mammar, "On the estimation of longitudinal dynamics of powered two-wheeled vehicles," in European Control Conference, 2015.

[9] G. Panzani, M. Corno, and S. Savaresi, "16th ifac symposium on system identification longitudinal velocity estimation in single-track vehicles," IFAC Proceedings Volumes, vol. 45, no. 16, pp. 1701 $1706,2012$.

[10] L. Nehaoua, D. Ichalal, H. Arioui, S. Mammar, and L. Fridman, "Lean and steering motorcycle dynamics reconstruction: An unknown-input HOSMO approach," in American Control Conference (ACC),, June 2013, pp. 2821-2826.

[11] D. Ichalal, H. Arioui, and S. Mammar, "Observer design for twowheeled vehicle: A Takagi-Sugeno approach with unmeasurable premise variables," in 19th Mediterranean Conference on Control Automation (MED),, June 2011, pp. 934-939.

[12] I. Boniolo, S. M. Savaresi, and M. Tanelli, "Lean angle estimation in two-wheeled vehicles with a reduced sensor configuration," in 2012 IEEE International Symposium on Circuits and Systems, May 2012, pp. 2573-2576.

[13] M. Corno, G. Panzani, and S. M. Savaresi, "Traction-control-oriented state estimation for motorcycles," Control Systems Technology, IEEE Transactions on, vol. 21, no. 6, pp. 2400-2407, 2013.

[14] M. E.-H. Dabladji, D. Ichalal, H. Arioui, and S. Mammar, "Unknowninput observer design for motorcycle lateral dynamics: Ts approach," Control Engineering Practice, vol. 54, pp. 12-26, 2016.

[15] L. Nehaoua, D. Ichalal, H. Arioui, S. Mammar, and L. Fridman, "An unknown-input HOSM approach to estimate lean and steering motorcycle dynamics," IEEE Transactions on Vehicular Technology, vol. 63, no. 7, pp. 3116-3127, Sept 2014.

[16] A. Teerhuis and S. Jansen, "Motorcycle state estimation for lateral dynamics," Vehicle System Dynamics, vol. 50, no. 8, pp. 1261-1276, 2012.

[17] D. Ichalal, H. Dabladji, H. Arioui, S. Mammar, and L. Nehaoua, "Observer design for motorcycle lean and steering dynamics estimation: A Takagi-Sugeno approach," in American Control Conference (ACC), June 2013, pp. 5654-5659.

[18] K. Tanaka and H. Wang, Fuzzy Control Systems Design and Analysis: A Linear Matrix Inequality Approach. John Wiley and Sons, 2001. 